No. 20, June. 1962

\title{
On the Double Oven Method of Mass Spectrometer for Relative Partial Pressure Measurements*
}

\author{
Minoru TsudA**
}

(Received February 25, 1962)

\begin{abstract}
The frit-type double oven method of mass spectrometer has been solved for molecular gas flow. The fragmentation coefficient and relative partial pressure are calculated for the monomer and dimer of alkali halides in the single component system. Simple formula for experiment is derived to determine relative partial pressure of monomer and dimer. In case of existence of monomer, dimer and trimer, relative partial pressures are also calculated.
\end{abstract}

\section{Introduction}

Concerning as mass spectrometric studies about the high temperature thermochemical property of substances ${ }^{1,2)}$, the double oven method was proposed ${ }^{3,4)}$ and used ${ }^{5)}$ in connection with a study of polymeric species in equilibrium with alkali halides ${ }^{6}$. From usual single oven measurements alone, it was impossible to determine the relative amounts of polymeric species, since the relative ionization cross-sections were unknown.

In the previous works ${ }^{3)}{ }^{5)}$, two ovens connected with a pipe freely established the same pressure. Then the vapor pressure in the upper oven was so limited as the mean free path of vapor should be larger than twenty times of the effusion hole diameter of the upper oven and smaller than the connected pipe diameter.

The existence of the lower and upper limits of the mean free path restricts the vapor pressure within the samll range or there exists no suitable pressure ragne for usual effusion hole and pipe diameters, and then there exists no suitable temperature range of the upper oven. On the other hand, two ovens can be connected with small size of openings or frit ${ }^{7)}$. In this case the mean free path of upper oven vapor needs at least large compared with twenty times of the diameter of the effusion hole, but there is no restriction on the upper limit of the mean free path. The mean free path of lower oven vapor needs large compated with twenty times of the diameter of the frit openings in order to maintain molecular flow, and there is also no restriction on the upper limit. Accordingly, suitable temperature range of the upper oven may be found for temperatures of the lower oven, so that both gas flows from the lower to the upper oven and from the upper oven to the ion source of mass spectrometer are molecular flows. Hence, the ratios of the ionization constants and fragmentation coefficient of polymeric species can be determined for single component system by the frit-type double oven method.

\section{Ratio of Ionization Constants for Monomer and Dimer}

Let us assume that only monomers and dimers exist and the lower oven contains the condensed

* This work was studied at Argonne National Laboratoty, Argonne, Ill., U.S.A.

**Mass Spectrometer Labotatory, Tokyo Institute of Technology (Ohokayama, Meguro-ku, Tokyo) 
phase at temperature $T_{1}$ and vapor pressure $P_{1}$, and that upper oven themperature $T_{2}$ is little higher than $T_{1}$ and upper oven pressure is $P_{2}$. Then upper oven temperature is changed to $T^{\prime}{ }_{2}$ little higher than $T_{2}$ at constant lower oven temperature. Let $N_{12}$ is the mole number of gas flow per unit time from the lower oven to the upper one, $N_{23}$ is the mole number of gas flow per unit time from the upper oven to outside of it, and each suffix $\mathrm{m}, \mathrm{d}, 1,2$, and 3 to characterize monomer, dimer, the lower oven, the upper oven and outside of the upper oven respectively.

From mass conservation law in the steady state following equations are obtained.

$$
\begin{aligned}
N_{m 12}+2 N_{d 12} & =N_{m 23}+2 N_{d 23} \\
& =N_{m 23}{ }^{\prime}+2 N_{d 23}{ }^{\prime}
\end{aligned}
$$

where $N^{\prime}$ represents the mole number of gas flow per unit time at uppe oven temperature $T_{2}{ }^{\prime}$ and constant lower oven temperature $T_{1}$. Considering well known quantity of molecular flow $Q^{*}$ is equal to $P V$ and $N R T$ where $V$ and $R$ are the volume of gas flow per unit time and gas constant respectively, $N$ can be written as follows,

$$
\begin{aligned}
& N_{m 23}=K_{23}\left(1 / T_{2} M_{m}\right)^{1 / 2}\left(P_{m 2}-P_{m 3}\right) \\
& N_{d 23}=K_{23}\left(1 / T_{2} M_{d}\right)^{1 / 2}\left(P_{d 2}-P_{d 3}\right) \\
& N_{m 23}{ }^{\prime}=K_{23}\left(1 / T_{2}{ }^{\prime} M_{m}\right)^{1 / 2}\left(P_{m 2}{ }^{\prime}-P_{m 3}{ }^{\prime}\right) \\
& N_{d 23}{ }^{\prime}=K_{23}\left(1 / T_{2}^{\prime} M_{d}\right)^{1 / 2}\left(P_{d 2}{ }^{\prime}-P_{d 3}{ }^{\prime}\right)
\end{aligned}
$$

where $K_{23}$ is a constant independent of the pressure, temperature and molecular weight of gases, but depend on the gas leak size. This Equation (3) is substituted in Equations (1) and (2). Considering $P_{2} \gg P_{3}$ and $2 M_{m}=M_{d}$ where $P_{m 2}, P_{m 3}, M_{m}$ and $M_{d}$ are monomer pressure in the upper oven and at outside of the upper oven, molecular weight of monomer and dimer respectively, one gets

$$
\left(1 / T_{2}\right)^{1 / 2}\left(P_{m 2}+\sqrt{2} P_{d 2}\right)=\left(1 / T_{2}{ }^{\prime}\right)^{1 / 2}\left(P_{m_{2}}{ }^{\prime}+\sqrt{2} P_{d 2}{ }^{\prime}\right) .
$$

It can be shown ${ }^{8)}$ from ideal gas theory that pressure $P_{i}$ of a specy in the Knudsen cell is related to the measured ion intensity $I_{i}$ by

$$
P_{i}=k_{i} I_{i} T
$$

where $k_{i}$ is a proportionality constant assumed independent of temperature, but dependent on such as the beam geometry, the ionization cross-section, and the collection and detection efficiencies.

Equation (4) plus four equations similar to (5) constitute five equations in six unknowns, the unknowns being four pressures and two proportionality constants. Thus one can solve for the ratio of any two pressures or for the ratio of constants, obtaining

$$
\begin{aligned}
& k_{d} / k_{m}=\left(I_{m}{ }^{\prime} T_{2}{ }^{1 / 2}-I_{m} T_{2}{ }^{1 / 2}\right) / \sqrt{2}\left(I_{d} T_{2}{ }^{1 / 2}-I_{d}{ }^{\prime} T_{2}{ }^{1 / 2}\right) \\
& P_{d} / P_{m}=k_{d} I_{d} / k_{m} I_{m} .
\end{aligned}
$$

If the temperature change is small compared with ion intensity changes one can cancel the temperature terms in Equation (6) assuming $T_{2}{ }^{1 / 2} \approx T_{2}{ }^{1 / 2 / * *}$.

Then the following equation is obtained.

$$
k_{d} / k_{m}=\left(I_{m}{ }^{\prime}-I_{m}\right) / \sqrt{2}\left(I_{d}-I_{d}{ }^{\prime}\right) .
$$

Ion currents $I^{\prime}{ }_{m}$ and $I_{w}$ in above formula can not be measured directly, because the fragment ions of the same mass come from dimer $I_{d}{ }^{\prime}$ and $I_{d}$. So the fragmentation coefficient should be determined ${ }^{4}$. When the temperature of the lower oven is raised up to $T_{1}{ }^{*}$, the vapor pressures in the lower oven increase and the vapor pressure $P_{m}, P_{d}$ in the upper oven increase to $P_{m}{ }^{*}, P_{d}{ }^{*}$. While the temperature $T_{2}$ of the upper oven remains constant, and following equilibrium is attained for the upper oven,

* $Q=K(T / M)^{1 / 2}\left(P_{2}-P_{3}\right)$ dyne. $\mathrm{cm} / \mathrm{sec}$.

** For alkali halides $1^{\circ}$ temperature change at $1000^{\circ} \mathrm{K}$, i.e. $0.1 \%$ temperature change causes about $3 \%$ ion intensity change experimentally. 
No. 20, June. 1962

M. Tsuda: On the Double oven Method of Mass

$$
P_{m}^{* 2} / P_{d}^{*}=P_{m}^{2} / P_{d}=K_{p}
$$

where $K_{p}$ is the equilibrium constant between dimer and monomer pressures at constant temperature. Ion currents $I_{d}^{*}$ and $I_{d}$, corresponding to dimer pressure $P_{d}^{*}$ and $P_{d}$, are represented as

$$
P_{d}^{*} / P_{d}=I_{d}^{*} / I_{d}=n \text {. }
$$

From Equations (7) and (8), the following Equation (9) is obtained.

$$
P_{m}^{*} / P_{m}\left(=I_{m}^{*} / I_{m}\right)=\sqrt{n} \text {. }
$$

The fragmentation coefficient $k$ is defined as follows

$$
\begin{aligned}
& I_{m}=I_{m d}-k \cdot I_{d} \\
& I_{m}{ }^{*}=I_{m d}{ }^{*}-k \cdot I_{d}{ }^{*} \\
& I_{m}{ }^{\prime}=I_{m d}{ }^{\prime}-k \cdot I_{d}{ }^{\prime}
\end{aligned}
$$

where suffix md refers to the total monomer ions from monomer molecules plus cracked one from dimers, and $I^{*}$ and $I$ refer to the ion currents at lower oven temperature $T^{*}{ }_{1}$ and $T_{1}$ respectively and upper oven temperature $T_{2}$,

The fragmentation coefficient $k$ is obtained from Equations (10) and (11), using Equations (8) and (9), as follows,

where

$$
k=\left(I_{m d} *-\sqrt{n} I_{m d}\right) / I_{d}(n-\sqrt{n})
$$

$$
n=I_{d}^{*} / I_{d} \text {. }
$$

Then, Equations (10) and (12) are substituted into Equation (6b), finally Equation (14) for $k_{d} / k_{m}$ is obtained as following relation,

where

$$
k_{d} / k_{m}=1 / \sqrt{2}\left\{\left(\Delta I_{m d} /-\Delta I_{d}\right)+k\right\}
$$

$$
\begin{aligned}
& \Delta I_{m d}=I_{m d}{ }^{\prime}-I_{m d} \\
& \Delta I_{d}=I_{d}{ }^{\prime}-I_{d} .
\end{aligned}
$$

Using the relation shown in Equation (13), the fragmentation coefficient $k$ can be experimentally determined by changing lower oven temperature at constant temperature of the upper oven. While, the ratio $I_{m d} /-I_{d}$ in Equation (14) can be experimentally determined from the following procedure. After that the thermal equlibrium was attained between the upper oven and its heating system, the heater resistance of the upper oven is slightly decreased and the resulted variation of the peak heights of the total monomer ion currents $I_{m d}$ and the dimer ion curents $I_{d}$ are recorded at the same time. The slope ratio of the peak heights caused by the transient temperature increase in few degrees of the upper oven gives $\Delta I_{m d} /-\Delta I_{d}$ shown in Equatoin (14). Consequently, one can determine the ratio of the proportionality constants $k_{d} / k_{m}$ using Equation (14).

\section{Ratios of Ionization Constants for Monomer, Dimer and Trimer}

Analogously, when there are not only monomer and dimer but trimer-the quantities concerned to trimer are denoted by the suffix $\mathrm{t}$ - the rations of the proportionality constatnts can be determined by changing upper oven temperatures from $T_{2}$ to $T_{2}{ }^{\prime}$ and $T_{2}^{\prime \prime}$. The following two molecular flow equations at each respective temperature are obtained,

$$
\left.\begin{array}{l}
N_{m 23}+2 N_{d 23}+3 N_{t 23}=N_{m 23}{ }^{\prime}+2 N_{d 23}{ }^{\prime}+3 N_{t 23}{ }^{\prime} \\
N_{m 23}+2 N_{d 23}+3 N_{t 23}=N_{m 23}{ }^{\prime \prime}+2 N_{d 23}{ }^{\prime \prime}+3 N_{t 23}{ }^{\prime \prime}
\end{array}\right\}
$$

where $N_{23}, N_{23}^{\prime}$ and $N_{23}{ }^{\prime \prime}$ denote the mole numbers of the molecular flow per unit time from the upper oven to outside of it at upper oven temperatures $T_{2}, T_{2}^{\prime}$ and $T_{2}^{\prime \prime}$, respectively. 'These mole 
numbers may be represented by equations silmilar to Equation (3), and each pressure may be represented by following nine equations,

$$
\left.\begin{array}{lll}
P_{m}=k_{m} I_{m} T & P_{m}{ }^{\prime}=k_{m} I_{m}{ }^{\prime} T^{\prime} & P_{m}{ }^{\prime \prime}=k_{m} I_{m}{ }^{\prime \prime} T^{\prime \prime} \\
P_{d}=k_{d} I_{d} T & P_{d}^{\prime}=k_{d} I_{d}{ }^{\prime} T^{\prime} & P_{d}{ }^{\prime \prime}=k_{d} I_{d}{ }^{\prime \prime} T^{\prime \prime} \\
P_{t}=k_{t} I_{t} T & P_{t}^{\prime}=k_{t} I_{t}{ }^{\prime} T^{\prime} & P_{t}^{\prime \prime}=k_{t} I_{t}{ }^{\prime \prime} T^{\prime \prime}
\end{array}\right\}
$$

where $P, P^{\prime}$ and $P^{\prime \prime}$ are upper oven pressures of each molecular specy corresponding to upper oven temperatures $T, T^{\prime}$ and $T^{\prime \prime}$. The equations similar to Equation (3) and concerned to $N_{23}, N_{23}{ }^{\prime}$ and $N_{23}{ }^{\prime \prime}$ are substituted into Equation (15), and eleven equations from Equations (15) and (16) are obtained with twelve unknowns which are nine pressures and three ionization constants. Thus one may solve for the ratios of the ionization constants or partial pressures from similar way as mentioned above for two kinds of molecular species,

$$
\begin{aligned}
& k_{d} / k_{m}=\left\{\left(I_{m} T_{2}{ }^{1 / 2}-I_{m}^{\prime} T_{2}^{\prime}{ }^{1 / 2}\right)\left(I_{t} T_{2}{ }^{1 / 2}-I_{t}{ }^{\prime \prime} T^{\prime \prime}{ }_{2}{ }^{1 / 2}\right)\right. \\
& \left.-\left(I_{m} T_{2}{ }^{1 / 2}-I_{m}^{\prime \prime} T^{\prime \prime}{ }_{2}{ }^{1 / 2}\right)\left(I_{t} T_{2}{ }^{1 / 2}-I_{t}^{\prime} T^{\prime}{ }_{2}{ }^{1 / 2}\right)\right\} / \\
& \sqrt{2}\left\{\left(I_{d} T_{2}{ }^{1 / 2}-I_{d}{ }^{\prime \prime} T_{2}{ }^{\prime \prime 1 / 2}\right)\left(I_{t} T_{2}{ }^{1 / 2}-I_{t}{ }^{\prime} T^{\prime}{ }^{1 / 2}\right)-\right\} \\
& \left.\left(I_{d} T_{2}{ }^{1 / 2}-I_{d}^{\prime} T^{\prime}{ }_{2}{ }^{1 / 2}\right)\left(I_{t} T_{2}{ }^{1 / 2}-I_{t}{ }^{\prime \prime} T_{2}{ }^{1 / 1 / 2}\right)\right\} \\
& P_{d} / P_{m}=k_{d} I_{d} / k_{m} I_{m} \\
& k_{t} / k_{m}=\left\{\left(I_{m} T_{2}{ }^{1 / 2}-I^{\prime}{ }_{m} T^{\prime}{ }_{2}{ }^{1 / 2}\right)\left(I_{d} T_{2}{ }^{1 / 2}-I_{d}{ }^{\prime \prime} T_{2}{ }^{1 / 2}\right)-\right. \\
& \left.\left(I_{m} T_{2}{ }^{1 / 2}-I_{m}{ }^{\prime \prime} T^{\prime \prime}{ }_{2}{ }^{1 / 2}\right)\left(I_{d} T_{2}{ }^{1 / 2}-I_{d}^{\prime} T^{\prime}{ }_{2}{ }^{1 / 2}\right)\right\} / \\
& \sqrt{3}\left\{\left(I_{t} T_{2}{ }^{1 / 2}-I_{t}{ }^{\prime \prime} T^{\prime \prime}{ }_{2}{ }^{1 / 2}\right)\left(I_{d} T_{2}{ }^{1 / 2}-I_{d}^{\prime} T^{\prime}{ }_{2}{ }^{1 / 2}\right)-\right. \\
& \left.\left(I_{t} T_{2}{ }^{1 / 2}-I_{t}{ }^{\prime} T^{\prime}{ }_{2}{ }^{1 / 2}\right)\left(I_{d} T_{2}{ }^{1 / 2}-I_{d}{ }^{\prime \prime} T^{\prime \prime}{ }_{2}{ }^{1 / 2}\right)\right\} \\
& P_{t} / P_{m}=k_{t} I_{t} / k_{m} I_{m}
\end{aligned}
$$

where $I, I^{\prime}$ and $I^{\prime \prime}$ are the ion currents corresponding to upper oven temperatures $T_{2}, T_{2}{ }^{\prime}$ and $T_{2}^{\prime \prime}$ respectively.

On the other hand, there is free connection between the lower oven and upper one, instead of the frit to obtain the pressure equilibrium in both ovens at constant lower oven pressure. The upper oven temperatures $T_{2}, T_{2}^{\prime}$ and $T_{2}^{\prime \prime}$ are slightly differed, following Equation (19) can be deduced instead of Equation (15),

$$
\left.\begin{array}{l}
P_{m}+P_{d}+P_{t}=P_{m}^{\prime}+P_{d}^{\prime}+-P_{t}^{\prime} \\
P_{m}+P_{d}+P_{t}=P_{m}^{\prime \prime}+P_{d}^{\prime \prime}+P_{t}^{\prime \prime} .
\end{array}\right\}
$$

Then the ratios of the proportionality constants are obtained from eleven equations from Equation (16) plus Equation (19) as follows.

$$
\left.\begin{array}{rl}
k_{d} / k_{m}= & \left\{\left(I_{m} T_{2}-I_{m}{ }^{\prime} T^{\prime}{ }_{2}\right)\left(I_{t} T_{2}-I_{t}{ }^{\prime \prime} T_{2}{ }^{\prime \prime}\right)-\left(I_{m} T_{2}-I_{m}{ }^{\prime \prime} T_{2}{ }^{\prime \prime}\right)\left(I_{t} T_{2}-I^{\prime}{ }_{t} T_{2}{ }^{\prime}\right)\right\} / \\
& \left\{\left(I_{d} T_{2}-I_{d}{ }^{\prime \prime} T_{2}{ }^{\prime \prime}\right)\left(I_{t} T_{2}-I_{t}{ }^{\prime} T_{2}{ }^{\prime}\right)-\left(I_{d} T_{2}-I_{d}{ }^{\prime} T_{2}{ }^{\prime}\right)\left(I_{t} T_{2}-I_{t}{ }^{\prime \prime} T_{2}{ }^{\prime \prime}\right)\right. \\
P_{d} / P_{m}= & k_{d} I_{d} / k_{m} I_{m} \\
k_{t} / k_{m}= & \left\{\left(I_{m} T_{2}-I_{m}{ }^{\prime} T_{2}{ }^{\prime}\right)\left(I_{d} T_{2}-I_{d}{ }^{\prime \prime} T_{2}{ }^{\prime \prime}\right)-\left(I_{m} T_{2}-I_{m}{ }^{\prime \prime} T_{2}{ }^{\prime \prime}\right)\left(I_{d} T_{2}-I_{d}{ }^{\prime} T^{\prime}{ }_{2}\right)\right\} / \\
& \left\{\left(I_{t} T_{2}-I_{t}{ }^{\prime \prime} T_{2}{ }^{\prime \prime}\right)\left(I_{d} T_{2}-I_{d}{ }^{\prime} T_{2}{ }^{\prime}\right)-\left(I_{t} T_{2}-I_{t}{ }^{\prime} T_{2}{ }^{\prime}\right)\left(I_{d} T_{2}-I_{d}{ }^{\prime \prime} T_{2}{ }^{\prime \prime}\right)\right\} \\
P_{t} / P_{m}= & k_{t} I_{t} / k_{m} I_{m}
\end{array}\right\}
$$

When the influence of trimer should be taken into consideration, Equations (17) and (18) or Equations (20) and (21) may be used in order to determine the ratios of the proportionality constants of monomer, dimer and trimer. 


\section{Discussion}

When simple formula (14) is used instead of Equation (6) to obtain the ratio of the ionization constants $k_{d} / k_{m}$, the slope ratio $\Delta I_{m d} /-\Delta I_{d}$ can be measured at one time. This eliminates or diminishes the experimental errors caused from variations of the frit size of the oven by contamination, ionization efficiency, background of ion source and detecting efficiency of ions in time interval on two separated measurements at different temperatures. For Equation (14) chemical equilibrium of dimer and monomer is unnecessary, but existence of the conservation law in steady state shown in Equations (1) and (2) is necessary. If steady state conditions (1) and (2) do not exist time in time, Equations (6), (10), (12) and (13) should be used in place of Equation (14) to obtain the ratio of the ionization constants $k_{d} / k_{m}$.

In case of monomer and dimer, the fragmentation coefficient is obtained by Equation (13). By similar way the fragmentation coefficients may be calculated for coexistence of monomer, dimer and trimer.

In Sections 2 and 3 calculations were made for one component system. By similar treatments, calculations for multi-component system may be done, but resulting equations may become considerably complicated.

Acknowledgments. - Author would like to thank Dr. W. A. Chupka and Dr. J. Berkowitz of Argonne National Laboratory for their generous advice and suggestion on the calculations of the double oven method.

\section{References}

1) J. Drowart and R.E. Honig, J. Chem. phys., 25, 581 (1956).

2) W.A. Chupka and M.G. Inghram, J. Phys. Chem., 59, 100 (1955).

3) T.A. Milne, J. Chem Phys., 28, 717 (1958).

4) L.N. Gorokhov, Ves. Mos. Univ., 6, 231 (1958).

5) P.A. Akishchin, L.N. Gorokhov and L.N. Sidorov, Zhur. Fiz. Khim., 33, 2822 (1959).

6) T.A. Milne, H.M. Klein and D. Cubicciotti, J. Chєm. Phys., 28, 718 (1958).

7) W.A. Chupka and et al., will be published.

8) R.E. Honig, J. Chem. Phys., 22, 126 (1954). 